\title{
Diacronie
}

Studi di Storia Contemporanea

$N^{\circ} 35,3 \mid 2018$

Gli strumenti di Clio

\section{Ebrei stranieri internati in Italia durante il periodo bellico}

Il sito e il database

Anna Pizzuti

\section{(2) OpenEdition \\ Journals}

Edizione digitale

URL: http://journals.openedition.org/diacronie/9285

DOI: $10.4000 /$ diacronie. 9285

ISSN: 2038-0925

\section{Editore}

Association culturelle Diacronie

Notizia bibliografica digitale

Anna Pizzuti, « Ebrei stranieri internati in Italia durante il periodo bellico », Diacronie [Online], №35,

3 | 2018, documento 17, Messo online il 29 septembre 2018, consultato il 22 avril 2019. URL : http:// journals.openedition.org/diacronie/9285; DOI : 10.4000/diacronie.9285 


\section{Diacronie}

Studi di Storia Contemporanea

$35,3 / 2018$

Gli strumenti di Clio: uomini, luoghi e teorie della storia dalla tradizione critica alla comunicazione digitale

\section{Ebrei stranieri internati in Italia durante il periodo bellico Il sito e il database}

\section{Anna PIZZUTI}

Per citare questo articolo:

PIZZUTI, Anna, «Ebrei stranieri internati in Italia durante il periodo bellico. Il sito e il database», Diacronie. Studi di Storia Contemporanea : Gli strumenti di Clio: uomini, luoghi e teorie della storia dalla tradizione critica alla comunicazione digitale, 35, 3/2018, 29/09/2018,

URL: < http://www.studistorici.com/2018/09/29/cegna_numero_35/ >

Diacronie Studi di Storia Contemporanea $\rightarrow$ http://www.diacronie.it

Rivista storica online. Uscita trimestrale.

redazione.diacronie@hotmail.it

Comitato di direzione: Naor Ben-Yehoyada - João Fábio Bertonha - Christopher Denis-Delacour - Maximiliano Fuentes Codera Anders Granås Kjøstvedt - John Paul Newman - Deborah Paci - Niccolò Pianciola - Spyridon Ploumidis - Wilko Graf Von Hardenberg

Comitato di redazione: Jacopo Bassi - Luca Bufarale - Gianluca Canè - Fausto Pietrancosta - Alessandro Salvador - Matteo Tomasoni - Luca Giuseppe Manenti

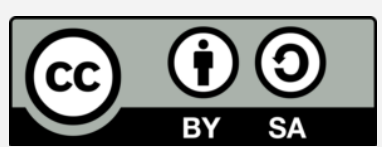

Diritti: gli articoli di Diacronie. Studi di Storia Contemporanea sono pubblicati sotto licenza Creative Commons 3.0. Possono essere riprodotti e modificati a patto di indicare eventuali modifiche dei contenuti, di riconoscere la paternità dell'opera e di condividerla allo stesso modo. La citazione di estratti è comunque sempre autorizzata, nei limiti previsti dalla legge. 


\section{7/ Ebrei stranieri internati in Italia durante il periodo bellico. Il sito e il database}

Anna PIZZUTI

Il saggio presenta una ricerca pubblicata on line nel 2009 ed in continuo aggiornamento. Ne illustra il contenuto, il metodo con cui viene condotta, le finalità e dimostra che, grazie al web, essa, attiva altre ricerche sullo stesso argomento e favorisce lo scambio e la condivisione di conoscenze.

\section{Gli ebrei stranieri}

Con l'espressione "ebrei stranieri" la burocrazia fascista indicava: a) gli ebrei di varia nazionalità che si erano stabiliti in Italia per motivi di lavoro, di studio o altro; b) ebrei profughi che speravano di poter partire verso altri paesi per sfuggire alle persecuzioni in atto nell'Europa centro orientale. Fino al 1936 il governo fascista consentì l'ingresso in Italia di ebrei stranieri, ma già nei mesi successivi iniziò la sorveglianza nei loro confronti. Nel settembre del 1938 fu revocata la cittadinanza italiana a quelli che l'avevano acquisita dopo il 1919, fu proibito l'ingresso dei profughi e fu stabilito per decreto che tutti avrebbero dovuto lasciare l'Italia. Nel giugno del 1940, con l'entrata in guerra dell'Italia quelli che non erano riusciti a partire furono internati in campi appositamente costruiti in alcune regioni del centro e del meridione. Per le donne fu previsto invece l'internamento in località non importanti dal punto di vista militare. Durante la guerra, nonostante i ripetuti ordini di respingimento, furono internati diversi gruppi di profughi, in maggioranza provenienti dalla Jugoslavia occupata. Ciascuno di questi passaggi fu accompagnato dalla produzione di una enorme quantità di documenti, che solo da qualche decennio sono diventati patrimonio della storiografia. 


\section{Come nasce il database}

L'idea di dedicare un sito web agli ebrei stranieri internati in Italia durante il periodo bellico con al centro un database che ne raccogliesse schematicamente le vicende, nasce durante le ricerche di archivio preparatorie alla stesura di un libro nel quale ho ricostruito la storia di un gruppo di internati nel paese di San Donato Valcomino, in provincia di Frosinone, tra il 1940 e il 1944.

La storia personale di ciascuno degli internati in quel piccolo paese, infatti, era riconducibile a storie più generali che avevano coinvolto gruppi più o meno numerosi di persone accomunate dalle diverse strategie di fuga alla ricerca di un rifugio per quanto precario. Ho iniziato, così, ad esaminare elenchi e fascicoli personali di singoli internati conservati sia presso l'Archivio Centrale dello Stato, passando poi a gli archivi di Stato delle varie province e gli archivi storici dei comuni ${ }^{1}$. C'erano migliaia di nomi, in quelle carte, moltissimi di persone ormai defunte, ma molti anche di persone la cui data di nascita portava a pensare che fossero ancora in vita e che probabilmente ignoravano il fatto che la loro esperienza vi fosse documentata. Ho quindi deciso di far riemergere dagli archivi i nomi che esse contenevano e segnare i passaggi principali del percorso compiuto da ciascuno durante gli anni della guerra conclusosi per molti con la deportazione.

\section{La pubblicazione online}

Tra il 2006 e il 2008 si venivano già strutturando diverse trasposizioni in rete di ricerche locali, relative in particolare alla provincia di Vicenza ${ }^{2}$. La mia intenzione era, invece, quella di allargare la ricerca a tutta l'Italia, perché di quelle vicende si cogliesse anche la valenza storica e si comprendesse fino a che punto e con quali modalità la macchina burocratica fascista fosse stata messa al servizio dei provvedimenti antiebraici. L'idea del database, inoltre, mi era stata fornita dalla conformazione stessa dei documenti che andavo consultando, soprattutto da quella degli elenchi, impostati quasi tutti sotto forma di tabelle. La stessa collocazione di quelli dai quali ho iniziato la raccolta dei dati, mi suggeriva il primo percorso storiografico sul quale lavorare. Mi riferisco alle liste ufficiali degli internati civili in Italia richieste dal Comitato Internazionale della Croce Rossa al Ministero degli Interni nel marzo del 1943. I fascicoli che le contengono sono

\footnotetext{
${ }^{1}$ Per le scoperte documentarie di partenza e per l'ampliamento delle ricerche, si veda il sito: PIZZUTI, Anna, «Presentazione del Database», in Ebrei stranieri internati in Italia durante il periodo bellico, URL:

< http://www.annapizzuti.it/public/presentazionedb.pdf > [consultato il 25 luglio 2018].

${ }^{2}$ Centro Studi sull'Internamento e la Deportazione dei Civili in Epoca Fascista, URL:

< http://www.dalrifugioallinganno.it > [consultato il 25 luglio 2018].
} 
suddivisi per province ed al loro interno sono raccolti anche gli elenchi degli ex internati che, dopo la liberazione, vi erano rimasti o che erano riusciti a raggiungerle fuggendo dai luoghi - in particolare da quelli situati nel nord Italia - nei quali erano rimasti bloccati dopo l'8 settembre. Era evidente che il confronto tra le due tipologie di elenchi avrebbe riportato alla luce i veri e propri itinerari verso la salvezza compiuti spesso anche attraversando le linee di combattimento. Altrettanto evidente era il fatto che lo strumento che avrebbe consentito la raccolta dei dati e la loro organizzazione doveva essere, necessariamente, un database. La quantità di informazioni che mi apprestavo a raccogliere escludeva, tuttavia, la possibilità di una pubblicazione cartacea, del tipo di quella inaugurata da Liliana Fargion Picciotto con il suo "Libro della memoria"'. In più con un libro non sarei riuscita a realizzare il work in progress che mi ripromettevo di condurre. La scelta della pubblicazione on line del database è stata, quindi, obbligata. Essa, tra l'altro avrebbe consentito: a) il contatto e lo scambio di informazioni con tutti coloro che avessero scoperto nel database il proprio nome o quello di familiari scomparso b) l'aggiornamento del database con i dati raccolti a seguito dell'estendersi della ricerca le cui fonti nel corso degli anni si sono notevolmente ampliate e che ancora oggi continua.

\section{I modelli, le fonti, il metodo}

Il database è stato messo on line nel 2009, all'interno del sito www.annapizzuti.it, il cui argomento è esplicitato nel sottotitolo "Ebrei stranieri internati in Italia durante il periodo bellico". All'epoca esistevano già diversi esempi di database on line contenenti informazioni sugli ebrei perseguitati prima e durante la Shoah, a partire da quello dello Yad Vashem insieme a quello presente sul sito del museo dell'olocausto di Washington che, a sua volta "pesca" in una serie di database realizzati da varie organizzazioni. Indispensabile per chi si occupa di approfondire in particolare le vicende degli ebrei residenti nella ex Jugoslavia negli anni della guerra è il database che contiene i nomi e informazioni sul destino di tutti gli iscritti alle Comunità ebraiche croate curato dalla storica Melita Svob. Per quanto riguarda l'Italia va citato il database che contiene nomi ed altre informazioni riguardanti gli intestatari di tutti i fascicoli personali degli ebrei stranieri internati in Italia conservati presso l'Archivio Centrale dello Stato, curato da Francesca Cappella ${ }^{4}$.

\footnotetext{
${ }^{3}$ PICCIOTTO, Liliana, Il libro della Memoria, Milano, Mursia, 2002.

${ }^{4}$ Per quanto riguarda lo Yad Vashem si veda, URL: < https://yvng.yadvashem.org/ >; per l'Holocaust Survivors and Victims Database si veda, URL: < https://www.ushmm.org/remember/the-holocaust-survivors-andvictims-resource-center/holocaust-survivors-and-victims-database > ; per il caso croato si veda, URL:

< http://www.cendo.hr/BazaZrtava.aspx >; infine, per il database Cappella, si veda, URL:

< http://www.cise.unipi.it/ebreistranieriitalia/ > [consultati il 25 agosto 2018].
} 
Fonti archivistiche e fonti reperibili in rete si sono quindi intersecate svolgendo ciascuna non solo il compito di pervenire all'esattezza ed alla completezza dei dati anagrafici indispensabili per la sicura identificazione di ciascuno degli internati, ma anche quello di un vero e proprio controllo reciproco. Ciascuna delle tipologie di fonti presenta comunque qualche limite. Il primo riguarda la grafia dei nomi trascritti negli elenchi conservati negli archivi che provoca la presenza di doppioni nel database, problema che risolvo grazie alla verifica della sovrapponibilità delle altre informazioni che li accompagnano. Speculare a questo è il problema delle omonimie che ho verificato soprattutto nei grandi database rinvenibili on line, in particolare quando i dati anagrafici non sono completi ed è quindi più facile cadere in errore. In questo caso il controllo risulta più difficoltoso, perché può essere risolto solo se si è in possesso di altre fonti validate. Un'altra difficoltà si incontra rispetto ai luoghi di nascita, quando questi non sono città molto note o facilmente identificabili. In questo caso l'inesattezza della grafia non influisce solo sulla completezza dell'identificazione, ma può rendere più difficoltosa la quantificazione del numero di profughi provenienti da una stessa nazione, dato che assume un importante ruolo nella ricostruzione delle politiche antiebraiche dei singoli Stati. Un contributo prezioso ai fini della correttezza dei dati, ad ogni modo, lo ha fornito il rapporto con i familiari o con le stesse persone i cui nomi compaiono nel database. Sono loro che, dopo essere arrivati anche casualmente sul mio sito, mi scrivono per correggere gli eventuali errori ed anche per fornire informazioni relative al percorso di salvezza realizzato dopo la liberazione.

\section{Le modalità di consultazione}

Non sono io a gestire la pubblicazione del sito, tranne che nella parte dell'admin nella quale inserisco i dati che vengo raccogliendo. Tuttavia la sua impostazione, a partire dalla stessa organizzazione del database, ed i vari percorsi di ricerca che esso doveva offrire sono stati concordati in modo da corrispondere agli obiettivi - anche storiografici - che mi ero proposta. La quantità delle chiavi di ricerca e il fatto che le informazioni relative a ciascun nome compaiano tutte nella pagina evitando così l'uso della barra di scorrimento orizzontale per visualizzarle tutte facilitano la consultazione. In più i meccanismi interni del sito fanno in modo che, chiamando un singolo nome, si possano visualizzare anche i nomi ed i percorsi di tutti gli eventuali componenti del nucleo familiare. L'ampliamento della ricerca ai gruppi di ebrei profughi internati in Italia anche durante la guerra, soprattutto quelli provenienti dalla Jugoslavia occupata, ha determinato la necessità di differenziare - tramite l'introduzione delle colonne intitolate "In Italia a" e "In Italia da" - gli ebrei stranieri internati al momento dell'entrata in guerra dell'Italia, da quelli arrivati successivamente. 
Il linguaggio php con il quale il sito è costruito consente, inoltre, di estrarre dallo stesso database le tabelle riassuntive raccolte nella sezione dei riepiloghi, mentre dall'area download è possibile scaricare in formato pdf elenchi specifici. Tabelle ed elenchi vengono aggiornati automaticamente. E' possibile in questo modo ottenere dati complessivi relativi al numero degli internati identificati, alle presenze nelle singole province, al numero di coloro che da esse vennero deportati, ai luoghi in cui essi si trovavano o che raggiunsero dopo la liberazione. Dalla sezione dei riepiloghi è possibile da poco accedere alla sezione dedicata alla nazionalità degli internati, risultato di una ricerca molto impegnativa ma - spero - significativa dal punto di vista storiografico. Come significativi, dallo stesso punto di vista, risultano tutti gli altri dati generali i quali restituiscono la dimensione complessiva dell'internamento degli ebrei stranieri in Italia.

\section{La rete, le reti, la condivisione}

La pubblicazione on line del database non è stata accompagnata da una vera e propria ricostruzione storica, bensì dalla trascrizione delle principali disposizioni - decreti, direttive, circolari - che, a partire dal 7 settembre del 1938, riguardarono gli ebrei stranieri presenti in Italia e da quella di alcune vicende esemplari che illustrassero la loro applicazione. Questa scelta ha fatto assumere al sito, fin dall'inizio, una funzione che potrebbe essere definita di servizio per chi desiderasse intraprendere ricerche sullo stesso argomento. Le fonti compaiono in sigla nell'ultima colonna, ma vengono esplicitate nella scheda collegata a ciascun nome, e raccolte in un elenco completo scaricabile dalla home page assumono carattere di servizio. L'insieme di queste informazioni diventa,così, una vera e propria guida alle tipologie di archivi, ai fondi nei quali generalmente vengono conservati i documenti riguardanti gli ebrei stranieri internati, ai database on line, alle ricerche svolte da altri studiosi. Ho potuto verificare la funzionalità di questi servizi attraverso i contatti che intrattengo in particolare con insegnanti che svolgono ricerche insieme ai propri alunni a partire dall'archivio storico del comune di residenza, ma anche con istituzioni, come gli stessi comuni, alcuni dei quali hanno scoperto proprio grazie al database, una parte di storia del proprio paese che era stata dimenticata. Accade anche che persone che avevano cercato nel database i propri familiari finiscano poi per estendere la ricerca a tutta la comunità dalla quale essi provenivano, contribuendo anche ad ampliare le tipologie di fonti documentali archivistiche o pubblicate on line. A circa nove anni dalla pubblicazione del sito debbo registrare, infine, il fatto che la rete ha a sua volta creato altre reti. Si è infatti sviluppata una vera e propria comunità di studiosi che ha prodotto a sua volta, oltre a numerosi libri, opuscoli, saggi, anche significative ed utilissime risorse on line $e^{5}$. Questa rete, ma anche ciascuna delle esperienze citate è

\footnotetext{
${ }^{5}$ Si veda, ad esempio, l'Archivio Maggioli Mazzoni, «Presentazione», in Archivio Maggioli Mazzoni. Ebrei
} 
caratterizzata dalla condivisione delle informazioni e delle stesse fonti documentali o di altro tipo. In questo modo diventa possibile accedere anche a documentazioni conservate in archivi di città diverse, o addirittura all'estero. Tuttavia l'elemento più significativo sul quale la rete si fonda risiede nel fatto che ad essere condiviso è il metodo con il quale le ricerche vengono condotte, e le finalità con le quali si intende riportare alla luce un particolare aspetto della persecuzione antiebraica per molti anni quasi ignorato, se non rimosso, dalla storiografia.

\section{Storia digitale o storia con il digitale?}

La raccolta di informazioni necessarie a riempire le troppe caselle del database che rimangono tuttora vuote continua ancora oggi, ma da qualche anno questa attività puramente compilativa ha generato una nuova sezione del sito denominata "Dal database alla storia". Ho infatti verificato che alcune delle informazioni che possono essere estratte dal database, relativamente soprattutto ai flussi di profughi internati durante la guerra, possano offrire un contributo non solo quantitativo, ma anche interpretativo delle politiche antiebraiche del fascismo, ad esempio nelle colonie - in particolare quella libica - e nei territori ex jugoslavi annessi o occupati dall'Italia tra il 6 aprile del1941 e l'8 settembre 1943 e dai tedeschi fino al maggio del 1945. Riguardo a quest'ultimo argomento va segnalato che le vicende successive alla fine della seconda guerra mondiale e l'impossibilità di accedere agli archivi della ex Jugoslavia che si è protratta fino al 1989 hanno notevolmente pesato sulla ricostruzione di diversi avvenimenti, e sulla loro interpretazione. Ricostruzione ed interpretazione che, in alcuni casi, si è fondata proprio su dati numerici non supportati dalle necessarie fonti.

Da questa constatazione sono nati alcuni brevi saggi nei quali il dato numerico costituito dalle cifre estraibili dal database viene messo a confronto con la documentazione archivistica soprattutto quella reperita nell'archivio di Stato di Fiume o - grazie ai contatti con altri storici nell'archivio del Museo Ebraico di Belgrado. Fulvio Cammarano, presidente della Sissco, intervenendo in una recente intervista sui processi generali di trasformazione in atto nel "mestiere dello storico" e quindi, anche sui nuovi strumenti divulgativi, si è soffermato sull'uso di

stranieri ed italiani internati nella provincia di Pesaro, URL: < http://www.archiviomaggiolimazzoni.it/ > [consultato il 30 luglio 2018]; e l'ampia ricerca sui campi fascisti di GIUSEPPINI, Andrea, «Home» in I campi fascisti. Dalle guerre in Africa alla Repubblica di Salò, URL: < www.campifascisti.it > [consultato il 30 luglio 2018]. Per quanto riguarda gli ultimi anni, vanno ricordate la messa in rete dell'aggiornamento del "Libro della memoria" citato: Fondazione CDEC, I nomi della Shoah italiana. Memoriale delle vittime della persecuzione antiebraica 1943-45, URL: < http://www.nomidellashoah.it/ > [consultaotil 30 luglio 2018]; si veda anche la trasposizione in rete dell'importante ricerca di FALK, Federico, «Ebrei a Fiume e Abbazia», in Le comunità israelitiche di Fiume e Abbazia tra le due guerre mondiali, URL: < https://www.bh.org.il/jewishspotlight/fiume/> [consultato il 4 agosto 2018] e, ultimo ma non meno importante, la ricerca di LORENTINI, Giuseppe, «Home», Documenti e Storia del Campo di Concentramento di Casoli (1940-1944), URL: $<$ https://www.campocasoli.org/ > [consultato il 4 agosto 2018]. 
quelli tecnologici, evidenziando come essi, pur apparendo «più in grado di soddisfare il bisogno del grande pubblico della storia come racconto più o meno avvincente, più disposti a dialogare [...] privilegino la memoria a scapito della comprensione». In un successivo passaggio dell'intervista, tuttavia, Cammarano, pur mantenendo la sua preoccupazione che la digitalizzazione abbia penalizzato un sapere complesso come la storia si chiede: «Ma perché non rovesciare il piano? Al di là dei canali tradizionali della comunicazione tra storici - seminari, workshop, convegni, ecc. perché non iniziare a riflettere seriamente su come possiamo piegare ai nostri scopi il carattere sempre più tecnologico della comunicazione?» ${ }^{6}$.

Per quanto riguarda il lavoro che ho presentato in questa sede, non posso essere io a giudicare se risponda o meno alle esigenze manifestate dal professor Cammarano. Mentre ne descrivevo sommariamente il contenuto ed il metodo, in effetti, mi veniva in mente di attribuirgli la definizione di "storia dal basso" perché svolto al di fuori dei luoghi accademici e perché aperto ai contributi più svariati purchè trovino riscontro in fonti validabili. Sono tuttavia portata a ritenere che esso possa collocarsi più che nel campo della storia digitale, in quello della storia con il digitale o, meglio, in quello di una tipologia di ricerca storica che, senza il digitale non sarebbe stato possibile realizzare.

\footnotetext{
${ }^{6}$ CAMMARANO, Fulvio, «Il passato in un presente che cambia. Conversando di storia con Fulvio Cammarano" in Storia e futuro, rivista di storia e storiografia on line, 46/2018, URL: $<$ http://storiaefuturo.eu/passato-un-presente-cambia-conversando-storia-fulvio-cammarano/> [consultato il 12 marzo 2018].
} 


\section{L'AUTORE}

Anna PIZZUTI è nata ad Alvito (FR) nel 1949. È stata insegnante di italiano e storia. È autrice di Vite di carta. Storie di ebrei stranieri internati dal fascismo, Roma, Donzelli, 2010. La sua ricerca sull'internamento degli ebrei stranieri internati in Italia durante il periodo bellico, corredata da un database costantemente aggiornato, è on line sul sito www.annapizzuti.it.

URL: < http://www.studistorici.com/progett/autori/\#Pizzuti > 